\title{
Fear of stinging insects in relation to state anxiety and trait anxiety in a group of patients with hymenoptera venom allergy undergoing immunotherapy
}

\author{
Agnieszka Woźniewicz¹, Ewa Szynkiewicz²,3, Krzysztof Pałgan³ ${ }^{3}$, Małgorzata Graczyk², Aneta Dowbór-Dzwonka², \\ Zbigniew Bartuzi ${ }^{3}$
}

${ }^{1}$ Department of Geriatrics, Faculty of Health Sciences, Collegium Medicum in Bydgoszcz, Nicolaus Copernicus University in Torun, Poland 2Department of Nursing in Internal Diseases, Collegium Medicum in Bydgoszcz, Nicolaus Copernicus University in Torun, Poland ${ }^{3}$ Chair and Clinic of Allergology, Clinical Immunology and Internal Diseases, University Hospital No. 2, Bydgoszcz, Poland

Adv Dermatol Allergol 2019; XXXVI (4): 472-477 DOI: https://doi.org/10.5114/ada.2018.78808

\begin{abstract}
Introduction: The level of fear of hymenoptera associated with stinging or envenomation may depend on situational factors and on trait anxiety characteristic of each patient.

Aim: To assess the relationship between fear of stinging insects on the one hand and state anxiety and trait anxiety on the other in a group of patients with hymenoptera venom allergy.

Material and methods: The study was conducted by the Department of Allergy, Clinical Immunology and Internal Diseases, Dr J. Biziel University Hospital No. 2 in Bydgoszcz, Poland. A total of 114 patients (71 women (63\%) and 43 men (37\%)) with hymenoptera venom allergy undergoing immunotherapy participated in the study. The StateTrait Anxiety Inventory (STAI) and a dedicated scale developed by the authors for this study were used.

Results: State anxiety was found to significantly $(p<0.05)$ correlate with anxiety in the present moment $(R=0.247)$, in a situation of real danger (appearance of an insect) $(R=0.223)$ and during the spring and summer seasons $(R=0.278)$. Trait anxiety was found to significantly $(p<0.05)$ correlate with anxiety before immunotherapy $(\mathrm{R}=0.261)$, in the present moment $(R=0.257)$, in a situation of real danger (appearance of an insect) $(R=0.254)$, and after an insect sting $(R=0.236)$. These were also weak correlations. The correlation between trait anxiety and anxiety during the spring and summer seasons was moderate $(R=0.331)$.

Conclusions: The levels of trait and state anxiety are associated with the magnitude of the fear of the danger. Respondents showed a higher level of anxiety in a situation of danger and after an insect sting.
\end{abstract}

Key words: anxiety, hymenoptera, immunotherapy.

\section{Introduction}

The level of fear of insects associated with stinging or envenomation may depend on situational factors and on trait anxiety characteristic of each patient. In most cases, contact with hymenoptera leads to local redness and swelling. These symptoms usually resolve within $2 \mathrm{~h}$. Another type of response to stinging or envenomation is the so-called large local reaction, which is characterised by swelling of more than $10 \mathrm{~cm}$ in diameter persisting for more than 24 h. Systemic allergic reactions are, however, the most dangerous responses to envenomation by hymenoptera. In allergic patients, these reactions may lead to anaphylactic shock and death. Severe anaphylactic reactions in the patients' past trigger anxiety reactions of varying severity [1].

The biological function of anxiety "the inseparable companion of every human being's life" is to warn of a perceivable, real danger. Sometimes the sense of danger is situated in a person's inner image of this danger. An anxiogenic factor, if it persists for too long, forces the body to remain in the state of readiness, vigilance, carefulness and may result in the destruction of certain individual resources. Various "definitions" of anxiety have been proposed, for example: "an emotion analogous to fear experienced without an external stimulus, without any imagin-

Address for correspondence: Agnieszka Woźniewicz, Department of Geriatrics, Faculty of Health Sciences, Collegium Medicum, Nicolaus Copernicus University, 9 M. Curie-Skłodowskiej St, 85-094 Bydgoszcz, Poland, phone: +48 886705764 ,

e-mail: awozniewicz@gmail.com

Received: 13.06.2018, accepted: 13.08.2018. 
able object, associated with somatic feelings", "negative emotional states and experiences associated with a subjective perception of events and states", "a basic mental experience informing about a threat; subjective but at the same time commonly manifested as fear, apprehension, restlessness, aggression, shame, anger, self-preservation instinct". The clinical form of anxiety is defined as "a syndrome of behavioural or psychological disorders of clinical relevance accompanied by somatic symptoms or impairment of at least one area of activity" [2].

Spielberger, the author of the tool for measuring the level of anxiety that has been used in the present study, defines state anxiety as being characterized by subjective, consciously perceived feelings of apprehension and tension accompanied by the related activation or stimulation of the autonomous nervous system [3]. State anxiety is manifested by a considerable variability in response to various threatening factors [4]. Trait anxiety, on the other hand, is a theoretical construct that denotes a motive or acquired behavioural disposition that makes an individual susceptible to perceiving a wide range of objectively harmless situations as threatening and responding to them with anxiety states which are disproportionately severe in relation to the magnitude of the objective danger. Spielberger emphasises the learned character of anxiety [3].

Individuals with a high level of trait anxiety do necessarily display a higher level of state anxiety on a permanent basis, compared with individuals with a low level of trait anxiety. These individuals will react with anxiety in highly threatening situations. For patients with hymenoptera venom allergy, the appearance of an insect may be considered a highly threatening situation. According to the authors of the STAI (State-Trait Anxiety Inventory), research shows that irrespective of the type of situation, a higher level of trait anxiety is associated with a higher level of state anxiety. The association between the level of trait anxiety and the magnitude of the response to the danger, i.e. state anxiety, is to some degree dependent on the nature of the danger $[3,4]$.

This study, therefore, addresses the association of fear of stinging insects with state and trait anxiety in a group of patients with hymenoptera venom allergy.

The results obtained using the anxiety scale developed by the authors of the present study for patients with insect venom anxiety should be considered in terms of state anxiety for each of the following situations separately: before immunotherapy, at the present moment (during this study), in a situation of real danger (appearance of an insect), after the insect sting, during the winter season, during the spring and summer seasons.

\section{Aim}

The study aimed to analyse the relationship between fear of stinging insects on the one hand and state anxi- ety and trait anxiety on the other in a group of patients with hymenoptera venom allergy.

\section{Material and methods}

The study was conducted by the Department of Allergy, Clinical Immunology and Internal Diseases, Dr J. Biziel University Hospital No. 2 in Bydgoszcz, Poland, in the years 2014-2015. The study was conducted in a total of 114 patients (71 women (63\%) and 43 men (37\%)) qualified for specific immunotherapy after severe anaphylactic reactions (grades III and IV according to the Mueller classification). Patients in their first, second, third, fourth and fifth year of immunotherapy accounted for, respectively, $33 \%$ (38), 18\% (21), 15\% (17), 13\% (15) and 17\% (19) of the respondents. Wasp venom allergy was diagnosed in 83 (73\%) and bee venom allergy in $31(27 \%)$ subjects. The respondents were divided into the following age groups: 18 to 35 years ( 29 subjects $(25.5 \%)$ ), 36 to 55 years ( 85 $(49 \%))$, and $56+$ years $(29(25.5 \%))$. More than half of them lived in urban areas $(66(58 \%))$, while the remainder lived in the country (46 (42\%)). A total of 79 (69\%) subjects were in a relationship, while 35 (31\%) declared being single. Most subjects had completed secondary (55 (48\%)) or higher (37 (33\%)) education and a minority (22 (19\%)) had only completed primary education. A total of 79 (69\%) declared being professionally active, while the remainder were retired or received disability allowance $(35$ (31\%)). Only several respondents described their financial situation as bad (4 (4\%)), with an overwhelming majority describing it as good (110 (96\%)).

The study was approved by the Bioethics Committee, Collegium Medicum, Nicolaus Copernicus University in Torun, Poland (resolution No KB 535/2013). A statistical analysis of the research material was conducted by hypothesis verification at the significance level of $p<0.05$.

The study was based on the diagnostic survey method using interview technique. Two instruments were used in the present study: the State-Trait Anxiety Inventory (STAI) [5] to determine state anxiety and trait anxiety, and a questionnaire developed by the authors for this study to measure anxiety in patients with insect venom allergy. The latter captured sociodemographic and clinical data.

The STAI is an instrument intended for examining anxiety defined as a transient and situational state of an individual, and as a relatively constant personality trait. This test was authored by Spielberger, Gorsuch and Lushene, and its Polish adaptation was prepared by Spielberger, Strelau, Tysarczyk and Wrześniewski [5]. The inventory consists of two subscales: X-1 and X-2. The $X-1$ subscale is used to measure state anxiety and the $X$-2 subscale to measure trait anxiety. The STAI asks 40 questions, 20 questions per subscale, to which the respondent chooses one of the four possible answers. The test reliability is satisfactory. Both scales are char- 
acterized by high internal consistency, with a slightly lower absolute stability, particularly in the case of the $\mathrm{X}-1$ scale. Construct validity for both scales has been confirmed in numerous studies.

The anxiety scale for patients with insect venom allergy is a graphical ratio scale where the subject, on continuous lines graded from 0 to 10, rates their anxiety in six aspects: before immunotherapy, at the present moment, in a situation of real danger (appearance of an insect), after the insect sting, during the winter season, and during the spring and summer seasons.

On this scale, 0 refers to the absence of anxiety, and 10 to the most severe anxiety experienced by the subject.

During the study each subject rated the severity of their anxiety using the above scale.

\section{Results}

The results confirmed the association between the level of anxiety measured by the STAI and the levels of anxiety before immunotherapy, in a situation of real danger (appearance of an insect), during the study, after the insect sting, and during the spring and summer seasons (Table 1).

State anxiety was found to significantly $(p<0.05)$ correlate with anxiety in the present moment $(R=0.247)$, in a situation of real danger (appearance of an insect) $(R=0.223)$ and during the spring and summer seasons $(R=0.278)$. These were weak correlations.

Trait anxiety was found to significantly $(p<0.05)$ correlate with anxiety before immunotherapy $(R=0.261)$, in the present moment $(R=0.257)$, in a situation of real danger (appearance of an insect) $(R=0.254)$, and after an insect sting $(R=0.236)$. These were also weak correlations. The correlation between trait anxiety and anxiety during the spring and summer seasons was moderate $(R=0.331)$.

\section{Difference in anxiety level in a situation of real danger (appearance of an insect) versus after an insect sting}

The Student $t$-test demonstrated a statistically significant difference in anxiety level in a situation of real danger (appearance of an insect) versus after an insect sting ( $p<0.001 ; t=-5.869)$. The subjects had a significantly higher level of anxiety after an insect sting than in a situation of real danger (appearance of an insect).
Difference in anxiety level during the spring and summer seasons versus the winter season

The Student $t$-test demonstrated a statistically significant difference in anxiety level between the spring and summer seasons and the winter season $(p<0.001$; $t=-15.291)$. The subjects had a significantly higher level of anxiety during the spring and summer seasons versus the winter season.

\section{Difference in anxiety during the study versus a situation of real danger (appearance of an insect)}

The Student $t$-test demonstrated a statistically significant difference in anxiety level during the study versus a situation of real danger (appearance of an insect) ( $p<$ $0.001 ; t=-6.845)$. The subjects had a significantly higher level of anxiety in a situation of real danger (appearance of an insect) than during the study.

\section{Difference in anxiety during the study versus after an insect sting}

The Student $t$-test demonstrated a statistically significant difference in anxiety level during the study versus after an insect sting ( $p<0.001 ; t=-10.846)$. The subjects had a significantly higher level of anxiety after an insect sting than during the study.

\section{Discussion}

Hymenoptera venom poses a significant risk to humans and can lead to serious anaphylactic reactions, shock and even death. The quantity and composition of the venom injected by specific hymenopterous insects vary. Notably, the effects of the venom are not always harmful. Beneficial effects of bee venom have been reported: antiviral, antibacterial, antiparasitic, suppressing the progression of certain tumour cells, and in rheumatoid diseases [6]. The present paper focuses on the unfavourable aspects affecting patients with hymenoptera venom allergy.

Severe hymenoptera venom allergy, which generates systemic anaphylactic reactions along with potential complications, is a rare population phenomenon. It is often the only health problem in otherwise healthy individuals. Apart from the episodes of exposure and their sequelae, individuals with this type of allergy do not experience any physical symptoms related to it. However,

Table 1. Levels of anxiety in specific situations

\begin{tabular}{|c|c|c|c|c|c|c|}
\hline Anxiety & $\begin{array}{c}\text { Before } \\
\text { immunotherapy }\end{array}$ & $\begin{array}{l}\text { In a situation } \\
\text { of danger }\end{array}$ & $\begin{array}{c}\text { During the } \\
\text { study }\end{array}$ & After stinging & $\begin{array}{c}\text { During winter } \\
\text { season }\end{array}$ & $\begin{array}{l}\text { During spring and } \\
\text { summer seasons }\end{array}$ \\
\hline State & - & 0.223 & 0.248 & - & - & 0.279 \\
\hline Trait & 0.262 & 0.255 & 0.257 & 0.236 & - & 0.331 \\
\hline
\end{tabular}


a personal experience of just one systemic reaction can bring about significant changes in the patient's psychology, modify their patterns of behaviour, and affect their social functioning (interactions with family and friends), professional functioning, and activity. According to the observations and studies so far, the major predictor of the above changes is anxiety. Anxiety is associated with the symptoms experienced during the reaction to an insect sting, with the feeling that one's life is in danger, a sudden loss of control over one's health, and subsequent anticipation of the above. The feeling of danger of being stung by an insect again is the main factor that decreases the patient's quality of life.

In their study published in 2016 and discussed below, the authors evaluated the fear of being stung by an insect again using a visual analogue scale (VAS), a scale commonly used for pain assessment and adapted for the purposes of their study. The subject was asked to rate the severity of their anxiety on a $10-\mathrm{cm}$ line, where 0 referred to the absence of anxiety, and 10 to the worst imaginable anxiety. The assessment was carried out at two time points: before and during specific immunotherapy. The study included patients with hymenoptera venom allergies diagnosed according to the European Academy of Allergy and Clinical Immunology (EAACI) standards (history, skin test results, specific lgE levels) who had a history of a grade III or IV reaction to an insect sting according to the Mueller classification. Induction of immunotherapy was performed according to the rush protocol. Anxiety level during venom immunotherapy (VIT) was always performed after achieving the target maintenance dose of $100 \mu \mathrm{g} / \mathrm{ml}$ of the venom.

The baseline anxiety level in this study was high and identical in both sexes at $8.8 \pm 0.9$. A strong correlation $(0.83 ; p<0.05)$ was found between baseline anxiety level and the time from the reaction to the insect sting: the shorter the time elapsed, the higher was the level of anxiety. The decrease in anxiety level on repeat assessment, after administration of a maintenance dose, was statistically significant $(p<0.05)$ : by an average of 5.8 points on the VAS scale. A larger change, of 6.3 points, was observed in men. The duration of treatment after initiation of VIT did not affect the secondarily assessed anxiety level. Similarly, no correlation was observed between the type of venom vaccine and the mean results on the VAS scale. It is reasonable to assume that the high baseline anxiety level was a motivating factor for initiating immunotherapy, although the long period of time that had elapsed from the systemic reaction to the initiation of VIT (3 months to 26 years) is rather surprising.

The authors also used in their study a two-item expectation of outcome questionnaire examining the patient's anticipation of the risk of again developing a severe systemic reaction after stinging (Question 1), and the risk of death associated with this reaction (Question 2) (Expectation of Outcome Questionnaire). The questionnaire was given to the subjects immediately after VIT initiation and after the maintenance dose was achieved. The VAS score correlated with the results obtained from the expectations questionnaire: the decrease in the level of anxiety during the treatment was accompanied by a change in the expected risk of a severe reaction reoccurring and death; as the estimated risk was lower, it could be anticipated that the feeling of safety increased [7].

In one of the studies, its authors compared the quality of life and the level of unease and depression in individuals with confirmed hymenoptera venom allergy and indications for specific immunotherapy with a venom vaccine (according to the EAACI recommendations) who refused such treatment and in those who agreed to receive it, during the treatment or immediately after its completion. The authors also attempted to identify the factors that prompted the patients considered eligible for VIT to give up this method of causal treatment. They used two questionnaires validated for Poland - the Vespid Allergy Quality of Life Questionnaire (VQLQ) and the Hospital Anxiety and Depression Scale (HADS) - having added questions about basic demographic data, severity of symptoms after stinging, duration of immunotherapy or the possible refusal to undergo immunotherapy along with the reason. Immunotherapy completers had been treated for 5 years and an average of 2.3 years elapsed since the completion of VIT. The patients undergoing VIT during the study had completed an average of 2.5 years of their therapy. Of the 63 subjects who eventually formed the study population, 23 gave up immunotherapy, and significantly more often $(p=0.03)$ these were individuals with bee venom allergy and those who had developed less severe symptoms after the insect sting (grade III according to the Mueller classification, $p=0.003)$. Their refusal was not affected by such demographic factors as sex, education or place of residence. Subjects who had not undergone VIT turned out to be more disciplined in carrying adrenaline pre-filled syringes with them during the insect season ( $84 \%$ vs. $59 \%$ of those undergoing immunotherapy), with the difference being statistically significant. It is reasonable to conclude that patients undergoing specific immunotherapy felt safer, and this was confirmed by the analysis of HADS scores. The level of anxiety was higher in subjects who had refused to undergo immunotherapy compared to those who had initiated it (7.2 \pm 4.0 vs. $4.4 \pm 3.5, p=0.03)$. Among the highest HADS scores analysed, as many as $70 \%(n=10)$ were obtained by those who had given up immunotherapy. The most common reasons for refusing to undergo VIT provided by the respondents were: difficulties in reconciling treatment with work (39\%), too long a distance to the immunotherapy centre (33\%), doubts as to the effectiveness of this form of treatment (11\%), and unwillingness to receive and anxiety about the injections (11\%). The quality of life analysis carried out for the group undergoing VIT and the group that refused it showed that the level of VQLQ in patients after treatment was signifi- 
cantly higher than in those not treated ( $4.6 \pm 1.5$ vs. 3.2 \pm 0.9 ), and this difference was statistically significant ( $p=$ 0.001). This level was also higher compared to the subjects who had started the therapy (VQLQ $=4.3 \pm 1.9)$ and those not treated $(\mathrm{VQLQ}=3.2 \pm 0.9$ ), although here the difference was not statistically significant $(p=0.06)$ [8].

A study was also conducted to assess the quality of life, anxiety level and depression in 71 patients with hymenoptera venom allergy (wasp, 52; bee, 19) during VIT initiation and at one year of treatment, and the influence of VIT on the quality of life. The second objective was to compare patients with improvement and with deterioration of the quality of life $(\mathrm{Q} O \mathrm{~L})$ during VIT in order to assess both the positive and the negative impact of VIT on the treatment results measured with QoL to be able to plan in the future the possible changes in treatment protocols that would increase treatment satisfaction. The third objective was to compare the quality of life scores between those with wasp venom allergy and those with bee venom allergy. The research tools used in this study included the VQLQ, HADS, STAI for adults and the Cantril ladder.

The baseline QoL score in subjects with wasp venom allergy was 3.7 and did not depend on the severity of the anaphylactic reaction. At one year of VIT, the VQLQ score increased to 4.3 and this change was statistically significant. Women rated their baseline quality of life lower, but the feeling of improvement at 1 year of immunotherapy was markedly pronounced. At 1 year, the VQLQ score clinically improved in $43 \%$ of the subjects, did not change in $41 \%$, and decreased in $15 \%$, with men predominating (females 3 vs. males 5).

Subjects with bee venom allergy had a significantly higher VQLQ score (4.50) before immunotherapy compared to subjects with vespid venom allergy, and it did not improve significantly (4.8) at 1 year of VIT. This is most likely due to the fact that the questionnaire had been developed with vespid venom allergy in mind.

In $10 \%$ of the subjects $(n=7)$, the HADS demonstrated depression (a score of more than 11), and in 32\% $(n=23)$, a high level of anxiety, which was more prevalent in women, although this difference was not statistically significant [9].

In another study, its authors assessed the quality of life and anxiety level in patients who had undergone a severe anaphylactic reaction, and evaluated their knowledge on venom allergy. A total of 61 patients (35 with wasp venom allergy and 26 with bee venom allergy) participated in this study. The study, on purpose, was conducted during the period of insect activity. The following instruments were used in this study: an allergy history interview, VQLQ, HADS and a self-rating sheet for anxiety/unease about subsequent envenomation (using a scale of 0 to 100) which also included additional questions about certain aspects of everyday life (relations with the immediate environment, impact on profession- al life) and a request to specify what their disease had changed the most in their life. The subjects were also tested on their knowledge about allergy, which was not only a research goal but also a utilitarian one (planning of patient education).

Subjects with wasp venom allergy demonstrated significantly greater deterioration of the quality of life compared to those with bee venom allergy, although this difference was not marked for trait anxiety. The mean VQLQ score was 4.57 in women and 5.29 in men, although the difference was not statistically significant, similarly to the fact that in the assessment of anxiety on a scale of 0 to 100 , the men rated it lower than did the women. As the authors confirmed, anxiety level gradually decreased in the course of VIT.

These results are consistent with the results of the present study, confirming the occurrence of anxiety and psychological responses having an impact on the functioning of patients with hymenoptera venom allergies.

\section{Conclusions}

Anxiety experienced by patients with hymenoptera venom allergy is an important factor affecting the functioning of this group of patients.

The levels of trait and state anxiety are associated with the magnitude of the fear of the danger. Individuals with a high level of trait anxiety, compared with individuals with a low level of trait anxiety, do not necessarily respond with an increased sense of anxiety in potential situations in which one is endangered by insect stinging and after stinging.

Living with hymenoptera venom allergy may be a source of anxiety and cause stress to the patients. When treating hymenoptera venom allergy, attention should be paid to the level of anxiety experienced by the patient and also to offering support in this aspect, according to the holistic approach to the patient.

\section{Conflict of interest}

The authors declare no conflict of interest.

\section{References}

1. Pałgan K. Postępowanie diagnostyczne i terapeutyczne u pacjentów uczulonych na jad owadów błonkoskrzydłych. Alergol Pol 2014; 1: 60-4.

2. Dosiak M. Lęk - aspekty medyczne. Wiad Lek 1997; 1-3: 21-7.

3. Spielberger CD. Theory and research on anxiety. In: Anxiety and behavior. Spielberger CD (eds). Academic Press, New York 1966.

4. Wrześniewski K, Sosnowski T, Jaworowska A, et al. Inwentarz Stanu i Cechy Lęku STAI. Pracownia Testów Psychologicznych, Warsaw 2006.

5. Muller HL. Diagnosis and treatment of insect sensitivity. J Astma Res 1966; 3: 331-3. 
6. Pałgan K, Bartuzi Z. Właściwości biologiczne jadu pszczół. Alerg Astma Immun 2009; 14: 17-9.

7. Mikulski D, Smorawska-Sabanty E, Kowalski ML. Jakość życia pacjentów z alergią na jad owadów błonkoskrzydłych odmawiających leczenia za pomocą immunoterapii swoistej. Alerg Astma Immun 2016; 21: 133-7.

8. Niedoszytko M, Majkowicz M, Chełmińska M, et al. Quality of life, anxiety, depression and satisfaction with life in patients treated with insect venom immunotherapy. Post Dermatol Alergol 2012; 29: 74-9.

9. Nowak N, Bazan-Socha S, Pulka G. et al. Evaluation of the quality of life in subjects with a history of severe anaphylactic reaction to the Hymenoptera venom. Pneum Alergol Pol 2015; 83: 352-8. 